\title{
VIOLENCIA Y CUERPO. ACTOS DE CIUDADANÍA*
}

\author{
Sergio Valenzuela Pulgar y Nicolás Ried Soto** \\ Universidad de Chile, Chile \\ omegalfa87@gmail.com, nicolasried@gmail.com
}

\begin{abstract}
RESUMEN: El problema de la servidumbre voluntaria, expuesto por Étienne de la Boétie como la obediencia de muchos a uno, ha sido un problema central de la filosofía política, particularmente de la discusión sobre el concepto ideología. Este texto versa sobre la servidumbre y sus formas; los objetos de éste son la manifestación de la libertad y, por contraparte, las manifestaciones del poder. La intención de la propuesta libertad/poder no es presentarla como una relación dicotómica, al contrario, mostrarla como un relato metodológico sobre el cual problematizaremos cierta discusión política que tiene como elemento central el concepto de ciudadanía. Teniendo en consideración la interpretación tradicional del poder, revisaremos los campos semánticos de los sistemas conceptuales de Hegel y Foucault.
\end{abstract}

Palabras clave: Servidumbre voluntaria, ciudadanía, violencia, cuerpo.

\section{VIOLENCE AND BODY. CITIZENSHIP ACTS}

\begin{abstract}
The problem of voluntary servitude, exposed by Étienne de la Boétie as the obedience of many to one ruler, has been a central problem in political philosophy, particularly in the discussion of the concept of ideology. This text discusses servitude and its forms, taking as its object the manifestation of freedom versus the manifestation of power. The intention behind using a framework of freedom/power is not to establish the relation between the two as dichotomous, but rather to display it as a methodological narrative, on which we will problematize a certain political discussion which has as its core element the concept of citizenship. Keeping in mind the traditional interpretation of power, we will also look over the semantic fields of the conceptual systems of Hegel and Foucault.
\end{abstract}

Keywords: Voluntary servitude, citizenship, violence, body. 


\section{PRÓlOGO}

Este texto versa sobre la servidumbre y sus formas; los objetos de éste son la manifestación de la libertad y, por contraparte, las manifestaciones del poder. La intención de la propuesta libertad/poder, no es presentarla como una relación dicotómica, al contrario, mostrarla como un relato metodológico sobre el cual problematizaremos cierta discusión política que tiene como elemento central el concepto de ciudadanía. Sin evadir la ubicación tradicional del poder (la jurídica, la estatal), se presentarán relecturas de algunos constructos conceptuales del campo semántico que constituye los problemas de la libertad y del poder.

Una comprensión clásica del poder, la de Hobbes, nos dice que "cualquiera cualidad que hace a un hombre amado o temido de otros, o la reputación de tal cualidad, es poder, porque constituye un medio de tener la asistencia y servicio de varios" ${ }^{1}$, es decir, el poder sería una cualidad que tiene como consecuencia un fundamento interior para la acción. Sin embargo, al revisar una de las aristas de esta concepción del poder evidenciamos que se manda a hacer por causa del amor o el temor. Es interesante, y nos fijaremos en ello, mostrar que la causa del temor, en este sentido, es la muerte violenta ${ }^{2}$.

El fundamento "muerte violenta" sostiene el sentimiento de "temor" como razón para la acción, y sobre ello, pensamos, que esta "razón para la acción” en su nivel más abstracto es corporal: la muerte violenta es violencia al cuerpo. La violencia, como uno de los elementos que constituyen una particular constelación de dispositivos de poder, se da existencia efectiva como mera violencia, o bien como violencia encausada a fines de justicia, o sea, el Derecho como violencia legítima, como fuerza. Es la violencia, bajo este entendimiento, el elemento desiderativo más básico que puede ser presentado como una buena razón para la acción de un otro.

Como tal, la violencia puede presentarse en diversos planos, siendo clásica la distinción entre alma y cuerpo: conocidas son las ideas de "violencia simbólica", "violencia psicológica", "violencia política", entre otras; lo es más la idea de "violencia corporal", siendo por este "sobresaber" existente acerca de la violencia, que de ella ya no se puede hablar sin precauciones: deja de existir un afuera de la violencia. Pensamos que, en un sentido gramaticalmente profundo, todo mecanismo de poder opera sobre las conductas, toda vez que la violencia opera sobre el cuerpo: constituye falsa distinción la del pensar y el hacer, también la del alma y el cuerpo, teniendo en cuenta interpretaciones ciertamente hegelianas, pero también foucaultianas.

Cuando el cuerpo de un sujeto hace, aun pensando que la causa es violencia no-corporal, dicha violencia opera en el plano de lo corporal, toda vez que se disciplina su cuerpo, en tanto lo que se restringe o impide son sus conductas: el relato de su libertad es interrumpido.

Новвеs, Thomas. El Leviatán. España, Madrid: Sarpe, 1984, pp. 99 - 100.

2 El problema de esta visión clásica del poder, consiste en que éste termina equiparándose a "muerte violenta", entendiendo que un fundamento interior para la acción no puede significar "poder", sino solamente fin o propósito. 


\section{EL CUERPO}

\section{I. HEGEL}

En el $\$ 48$ de la "Filosofía del Derecho", Hegel analiza la relación entre la libertad y el cuerpo. Encontramos allí una triple relación: el cuerpo-libertad para el Espíritu, el cuerpo-libertad para los otros, y el cuerpo-libertad para mí. El cuerpo es el instrumento de la libertad, desde la perspectiva de la exteriorización y consecuente existencia de la voluntad libre. Dice Hegel: "El cuerpo no es adecuado al espíritu por ser una existencia inmediata; para que sea su órgano dócil y su medio animado, es necesario que éste lo tome en posesión. Pero para los otros soy esencialmente libre en mi cuerpo tal como lo poseo inmediatamente." ${ }^{3}$

El cuerpo es necesario para la existencia de mi libertad, es además el signo para los otros de que soy libre y, asimismo, el medio por el cual el espíritu se pone subjetivamente en el Mundo. "Sólo porque soy viviente como libre en un cuerpo, no se debe abusar de esta existencia viviente hasta hacerla una bestia de carga". ${ }^{4}$ No es que el cuerpo sea sólo el medio entre la libertad (abstracta) y el hacer (la libertad efectiva), sino que la relación está dada por la necesaria indistinción que se requiere al hablar de la libertad y el hacer: el hacer corporal es el hacerse de la libertad. "Aun encadenado puedo ser libre gracias a mi voluntad de separar de mí las sensaciones particulares de tal situación. Para otros, en cambio, sólo soy libre si esa existencia corpórea lo es". 5

La forma de la libertad descrita en la primera parte de la cita anterior corresponde a una libertad imperfecta, es la libertad del estoico que presenta Hegel en la "Fenomenología del Espíritu”, es decir, es la libertad de una autoconciencia que nunca se reconciliará consigo misma.

El símbolo de la bestia de carga refiere a un cuerpo no-libre, a un sujeto deconstituido, desprovisto de su libertad, un siervo. Siendo equivalente a la noción foucaultiana de que no existe libertad sin posibilidad de resistencia, toda vez que un cuerpo que no puede resistir tampoco puede ser libre: una bestia de carga es un mero instrumento, lo radicalmente no-libre. La bestia de carga es nula en términos de resistencia.

La relación entre existencia y cuerpo para Hegel, en términos en que no forman una dicotomía, establecen las bases del punto de indiferenciación entre el ser y el hacer, siendo la visión dualista de lo moral sustento de la servidumbre. Sin libertad del cuerpo, la libertad del alma no es representada, ni mucho menos presentada. Hegel mismo lo dice en términos de efectuación de la libertad subjetiva: "[e]n lo viviente, lo singular no existe como parte sino como órgano en el que está presente lo universal como tal, por lo cual el asesinato no lesiona una porción

\footnotetext{
3 Hegel, G. W. F. Principios de la Filosofía del Derecho o Derecho Natural y Ciencia Política. Barcelona, España: Edhasa, 1999, p. $132, \$ 48$.

4 Ibid.

5 Cordua, Carla. Explicación sucinta de la Filosofia del Derecho de Hegel. Santa Fe de Bogotá, Colombia: Temis, 1992, p. 47 , \$48.
} 
de carne, algo singular, sino la vida misma" ${ }^{\text {; }}$ esto no quiere solamente decir que al vulnerar lo singular se vulnera eo ipso lo universal, sino también que para vulnerar lo universal debe pasarse a través de lo singular. Para vulnerar el ser, la vida, debemos destruir un ser o una vida en particular; para vulnerar una existencia, debe violentarse un cuerpo.

Hegel en la "Fenomenología del Espíritu" expone a la autoconciencia como una actividad, pero no como una que gira sobre sí misma; al contrario, la autoconciencia es una actividad en tanto "reflexión, que desde el ser del mundo sensible y percibido, es esencialmente el retorno desde el ser otro" 7 . En este momento del devenir de la conciencia, ya no estamos en la mera certeza de sí, sino que la autoconciencia necesita de otra para poder encontrar su satisfacción y por tanto, debe existir para otro y mediarse en él.

Sin caer en una repetición tautológica de nuestro autor, la respuesta de la autoconciencia a la otredad se corresponde a una relación simétrica de mutua mediación en el movimiento del reconocimiento: "[e]ste movimiento de la autoconciencia en su relación con otra autoconciencia se representa, empero, de este modo, como el hacer de la una; pero este hacer de la una tiene él mismo la doble significación de ser tanto su hacer como el hacer de la otra." cuenta que el "se representa" de lo dicho quiere decir que no se presenta como tal, pues "[c]ada una de ellas ve a la otra hacer lo mismo que ella hace [...] el hacer unilateral sería ocioso, ya que lo que ha de suceder sólo puede lograrse por la acción de ambas" ". Este comportamiento especular de la relación expuesta nos da luces de lo que ocurre efectivamente: las autoconciencias "se reconocen como reconociéndose mutuamente" 10 .

Cabe preguntarse por el elemento que ocasiona la disolución del equilibrio de los haceres de estas autoconciencias. En términos del "hacer duplicado" de éstas, cada una tiende a la muerte de la otra. Es, por tanto, el temor a la muerte violenta que se da en esa lucha en que cada una comprueba a la otra lo que ocasiona el reconocimiento.

Se ha pensado separadamente el ser del hacer, pero ello no da cuenta de lo que aparece como servidumbre: la servidumbre es una creencia en que mis actos están necesariamente ligados a una esencia. Si en el hacer está la perspectiva sobre la cual hablaremos de la libertad, la violencia opera en las restricciones u obligaciones de ese hacer: actuar creyendo que esos actos son el producto de una esencia. El lugar en el cual el Yo hace es el Mundo, no en el topus uranus.

La problematización anterior se presenta como una forma de la libertad, mostrada en función de las posibilidades del hacer y cuáles son las acciones liberadoras: el problema de la

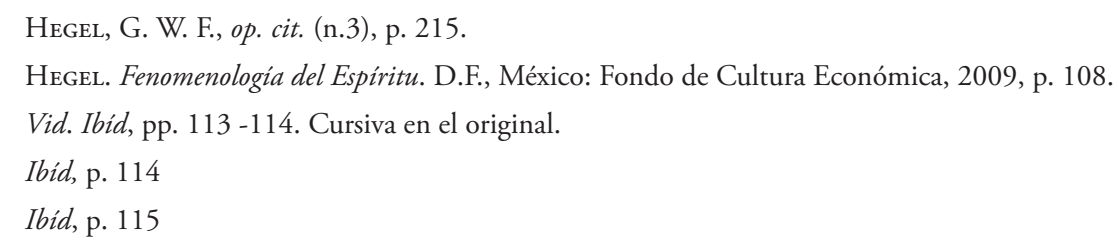


Identidad, en palabras de Kojève: "la Identidad, es el ser natural, por lo tanto el del hombre, es decir, todo lo que él ha recibido como dato fijo, su 'carácter' innato. Pero el hombre puede negar la naturaleza y su propia naturaleza: puede actuar. La Negatividad es la libertad (la acción libre o liberadora) la posibilidad que el hombre tiene de trascender su naturaleza; eso es lo que hay de propiamente humano en el Hombre"11.

La naturalización de conductas es la violencia al cuerpo de la que hablamos. Si existe algo como la "naturaleza humana", ella está dada por las infinitas posibilidades corporales del hacer, por negarse a sí misma en cada una de sus determinaciones.

\subsection{Foucault}

El problema prístino de la filosofía, estructurado tradicionalmente en la pregunta “¿quién soy?”, se traslada al problema político de la pregunta “¿en qué puedo convertirme?”, no es el problema de los límites de la libertad, sino de mis límites. Michel Foucault reconstruye el concepto de poder desde las posibilidades de resistirlo, abandonando un análisis unidireccional, estructurando una idea múltiple del mismo. Así, es conocida la idea de poder presentada en el primer tomo de la "Historia de la Sexualidad. La voluntad del saber": "El poder [...] es el nombre que se presta a una situación estratégica compleja en una sociedad dada"12.

Desde Foucault, no se puede hablar del poder, precisamente porque funciona de una manera discursiva y los individuos forman parte de ese discurso: si se pudiera aprehender el poder y definirlo, sería mera ilusión. El poder viene de todas partes y fluye por los cuerpos, regula los discursos y determina lo verdadero. El discurso es la verdad impuesta por el poder, que funciona mediante prohibiciones y normas. Mostrar cuáles son los límites sobre los que podemos comprehender esta concepción del poder es un dilema, aunque un esfuerzo útil consiste en establecer los límites negativos del poder, como también las diferentes manifestaciones conceptuales afirmativas que el propio Foucault organiza en su obra.

El primer tomo de la "Historia de la sexualidad", para Foucault, siempre se situó en el lugar de una base, organizando el sistema que ocuparía en la escritura de su Historia de la Sexualidad: el marco teórico necesitaba de una reestructuración de los mecanismos de poder ${ }^{13}$. Una primera silueta sobre el poder, en ese sentido, está dada por las negaciones del mismo, siendo un análisis interesante el que podemos obtener desde las negaciones que el mismo autor realiza sobre su concepción sobre el poder, debido a su relación con las prohibiciones y los silencios: "Uno sabe que no tiene derecho a decirlo todo, que no se puede hablar de todo en

Kojève, A. La dialéctica del amo y el esclavo en Hegel. Buenos Aires, Argentina: La Pléyade, 1985, p. 68.

12 Foucault, Michel. Historia de la sexualidad. La voluntad del saber. Buenos Aires, Argentina: Ediciones Siglo XXI editorial, 2008, vol. 1, p. 89.

13 Foucault, Michel. "Las relaciones de poder penetran en los cuerpos”; en su: Microfísica del poder. Madrid, Espańa: La Piqueta, tercera edición, 1992, p. 160. 
cualquier circunstancia, que cualquiera, en fin, no puede hablar de cualquier cosa"14. La posición del discurso foucaultiano necesita ser revelada, pero no puede ser revelada, ya que una definición del poder no permitiría en él la estructuración de un lugar de lucha en que, conceptualmente, pueda resistirse: una definición del poder es cristalizar y volver inerte la situación de poder que tal ejercicio presenta. Por ello, el ejercicio conceptual relevante en ese sentido es definir ciertos límites del poder, reconocer sus hitos externos y observarlos desde dentro, por ejemplo, desde las negaciones del poder. A modo de muestra, un conjunto de enunciados de lo que no constituye poder, según Foucault: No supone una situación binaria dominadores/ dominados ${ }^{15}$. No es un sistema general de dominación de un grupo sobre otro ${ }^{16}$. No obedece a la forma hegeliana de dialéctica ${ }^{17}$. No es unidimensional ${ }^{18}$, es múltiple. No es represión ${ }^{19}$, no es ideología ${ }^{20}$. No lo engloba todo: viene de todas partes ${ }^{21}$. No es exterior a otro tipo de relaciones $^{22}$. No actúa, necesariamente, a través de la violencia ${ }^{23}$. No opera, primeramente, sobre el alma: opera sobre el cuerpo ${ }^{24}$.

Lo destacable de esta lista, para estos efectos, es la operación que el poder realiza como fluido sobre el cuerpo, lo cual en su concepto técnico, Foucault bautiza como "anatomo-política": el entendimiento del cuerpo humano como máquina ${ }^{25}$, o si se quiere, como una bestia de carga. "El individuo es también, por lo tanto, en la medida en que se convierte en una máquina productiva y en una unidad reproductiva o consumidora de sexo". ${ }^{26}$

La anatomo-política opera en el nivel de la normalización, o sea, del disciplinamiento del cuerpo. En relación con ello, el poder opera sobre las conductas corporales, en lo micro: micro-disciplinas.

Siendo en este punto donde podemos reconocer la originalidad del argumento que reconoce que lo macro es una ilusión, pues en un sentido fantasmático el Capitalismo es indestructible: nos dice "si pretende criticar el sistema, proponga un sistema nuevo". Lo anterior le entre-

\footnotetext{
14 Foucault, Michel. El orden del discurso. Barcelona, España: Tusquets, 2002, p.14.

Foucault, Michel, op. cit. (n.12), p. 90.

Ibid, p. 88

Foucault, Michel. "Poder-cuerpo"; en su: Microfísica del poder. Madrid, España: La Piqueta, tercera edición, 1992, p. 106.

18 Godoy, Óscar. "Analítica del poder: Michel Foucault”, en Estudios Públicos No 40., Santiago, Chile: Centro de Estudios Públicos, primavera 1990, p. 113

Foucault, Michel, op. cit. (n.13), pp. 108-109.

Ibid.

1 Foucault, Michel, op. cit. (n.12), p. 89.

Ibid.

23 Deleuze, Gilles. Foucault. Barcelona España: Paidós, 2007, p. 53.

24 Foucault, Michel, op. cit. (n.13), p. 107.

25 Foucault, Michel, op. cit. (n.12), p. 131.

26 Huicı, Vicente. Espacio, tiempo y sociedad. Madrid, España: Akal, 2007, p. 73.
} 
ga un velo de acero transparente al Capitalismo, en vista de que las relaciones son disciplinantes en lo micro. Comprender el poder como una situación micro-política es el argumento sobre el cual se funda una teoría de la resistencia desde la performance; lo contrario, significa presuponer una verdad del cuerpo, en palabras de Deleuze: "El poder más que reprimir 'produce realidad', y más que ideologizar, más que abstraer u ocultar, produce verdad”. ${ }^{27}$

\section{LA VIOLENCIA}

Toda violencia es corporal. El discurso funciona en relación con una determinada dicotomía: lo normal y lo anormal. Tal relación funciona de manera binaria, dicotómica: lo que no funciona en el plano de lo normal deviene anormal, y viceversa. Tal relación, por tanto, se construye desde la exclusión de lo otro: lo normal encuentra sus límites en lo anormal, tal como lo anormal se ve limitado por lo normal.

En el proyecto de una reconceptuación de lo político desde la subversión, buscamos destacar las posibilidades de una sistemática degeneración de la mencionada relación normal/anormal. Así, se convierte en necesario mostrar un sistema de conductas que escape de la violencia ejercida por la dicotomía, en términos de la clasificación.

"Clasificación" comporta un concepto técnico: es la violencia corporal ejercida desde las redes discursivas, por las que fluye el poder y cae sobre los cuerpos; es decir, la naturalización de los cuerpos es una relación violenta, el afán de encasillar al cuerpo y sus funciones. Con ello nos referimos a la persistencia de la pregunta totalizante de la filosofía: “¿quién soy?”, siendo precisamente nuestra idea la cuestión contraria: la revelación de aquello en lo que puedo convertirme. La naturalización en este sentido se presenta como la forma en que los discursos sólo pueden decir un elemento de nosotros a la vez: es mujer, es comunista, es loco, es pobre, es homosexual, es judío, es negro, es ciudadano. Se excluyen de esta manera las situaciones de nuestra vida, de modo tal que se nos totaliza en un enunciado equivalente a tratarnos como "lo macro", toda vez que ello no coincide con una interpretación de lo que estamos actualmente siendo. La naturalización no da cuenta de la actualización del Yo, sólo se refiere eternamente a una idea preexistente sobre el Yo.

Sobre lo anterior, la búsqueda política está dirigida a las posiciones en las cuales no podemos ser clasificados, es decir, cuando el molde normal/anormal no puede operar sobre nosotros: escapar a lo normal sin caer en lo anormal y escapar de lo anormal sin caer en lo normal. Moverse entre las identidades, escapando a la Identidad.

27 Deleuze, Gilles, op. cit. (n.23), p. 55. 


\section{ACTOS CIUDADANOS}

La problematización teórica anterior sirve para presentar el problema político de la ciudadanía y la representación. Como demostramos desde Hegel, toda vez que la violencia es corporal, la violencia que conlleva la eliminación del individuo como político, es también violencia al cuerpo y un problema anatomo-político. Siempre que se anula al individuo en todos sus aspectos políticos y se le considera contra-fácticamente como ser político, existe violencia, siendo la violencia el elemento fundamental de la organización misma de la comunidad.

El reconocimiento que un gobierno, y los dispositivos de poder en general, objetivan sobre el individuo se da en términos de mecanismo universalizador del cuerpo, imponiendo una verdad sobre él y anulándolo funcionalmente: haciendo que sus actos coincidan con una esencia, una identidad. El quid, por tanto, es que el sistema ciudadano de la democracia es inexistente como sistema político.

El sistema democrático considera la ciudadanía como forma de acceso del individuo a la política, la participación en la práctica de la polis. La ciudadanía en nuestro sistema jurídicopolítico se presenta como un requisito para la participación y una vez obtenido se concretiza como mera representación: el ciudadano (quien cumple con los requisitos exigidos) es representado por el gobierno. Se da un enmarañado de ficciones: el sujeto actúa mediatamente, primero porque elige mediante el voto al gobierno, y segundo porque se presenta una identidad entre el gobierno y el sujeto.

Analizar las ficciones anteriores desde la propuesta teórica de Giorgio Agamben puede servirnos para reconocer por qué la democracia sólo da una ilusión de ciudadanía, la cual en su gramática profunda no existe. Agamben se refiere al concepto de "ejemplo" como una forma de inclusión por exclusión, es decir, aquel elemento de un conjunto que queda incluido en el mismo, precisamente porque no forma parte de él en tanto que es un ejemplo ${ }^{28}$. Así, no se puede decir de un elemento único de un conjunto que puede presentarse como un ejemplo, ya que si así fuera, el conjunto no tendría elementos y sería en sí mismo un ejemplo de nada: cuando la ciudadanía es definida como una actividad, revisamos que el conjunto de actos ciudadanos se reduce a la elección de autoridades políticas mediante el sufragio, por lo que los actos ciudadanos se reducen a su ejemplo y la ciudadanía, por tanto, se convierte en aquel elemento que se identifica con su conjunto. El significante ciudadanía, en ese sentido, deja de ser un correlato de su significado.

Ante tal dilema, el de una ciudadanía reducida a su inactividad, requiere de una forma de comprender lo ciudadano desde una óptica emancipatoria y subversiva, pasar desde la universalización de lo ciudadano a una práctica de lo ciudadano, un lenguaje público de la ciudadanía. Siguiendo la tesis de la disolución entre el ser y el hacer, la ciudadanía nominal se presenta

28 Agamben, Giorgio. Homo sacer. Valencia, España: Pre-textos, 2010, p. 35. 
como una no-ciudadanía, en tanto es un mero ser, sin tener en consideración el "hacer ciudadano". La asociación a una identidad no tiene sentido si el sujeto solamente adscribe a ella: ser miembro de un club sirve cuando el club realiza alguna actividad y hasta que la actividad acaba; el problema es entender la ciudadanía como a un club, porque las actividades de la polis no terminan, siempre vivimos en ella y estamos construyéndola.

\section{EPÍLOGO}

El poder, como situación estratégica compleja, como mecanismo de conducción de conductas y como actividad, opera sobre los cuerpos. Es el cuerpo el locus en el cual la violencia aparece y se desvanece, se hace una con el cuerpo violentado, transformándolo y transformándose: el cuerpo violentado no es igual tras la violencia, como tampoco lo es la violencia misma.

Desde Foucault, se entiende al poder como una entidad que fluye por los cuerpos, sin poder ser identificada o aprehendida, ya que no es un objeto ni un lugar: el poder de gobierno, la represión y la ideología son velos que cubren otro tipo de relaciones de poder. Los dispositivos de poder normalizan y serializan, hacen máquinas con hombres.

Sobre una lectura de Hegel, entendemos que toda violencia hecha a mi cuerpo es violencia hecha a mí, suponiéndose una determinada estructura exterior de la libertad que contempla la negación de ser una bestia de carga, la resistencia a ser una máquina.

Es sobre estas propuestas teóricas que entendemos que la participación ciudadana está maldita desde la democracia de la representación. Esta forma de contemplar la ciudadanía funciona como reductor del ciudadano a un mero accidente del mismo sistema político, ya que no está éste al servicio de los sujetos, sino al contrario: la actividad del sufragio es la única instancia de reconocimiento entre el participante y la práctica; la democracia persiste, pero el individuo existe intermitentemente.

Pensar la ciudadanía sin requisitos y no como una característica naturalizada es entender actos ciudadanos, es decir, una polis orgánica que asuma ciertas maneras de participación. No entender que se alcanza la ciudadanía, sino que se activa con formas de participación: la socialización de la ciudadanía no opera en cuanto se reproduce el sistema de inactividad en todas las esferas de lo social, sino cuando lo social opera en lo ciudadano.

La ciudadanía, de ser un estado que se obtiene y se pierde mediante el acto de desafío a las reglas de lo social (haber sido penado por pena aflictiva), pasa a estar radicalmente incorporada en el individuo: la radical comprensión de que los límites de lo humano coinciden con los límites de lo ciudadano. El comportamiento de un ciudadano es idéntico al comportamiento de un ser humano, ya que la no existencia en lo público es la conversión en una bestia de carga, un autómata que no puede sino cumplir las funciones básicas de la supervivencia: es la elección por la no reflexividad radical. 
No nos es posible definir las formas en las cuales se presentará esta actividad de lo ciudadano, ya que su definición importaría un retorno a la cristalización de lo ciudadano. La práctica liberadora es la práctica de buscar dicha práctica: es la meta-práctica de lo ciudadano.

\section{REFERENCIAS BIBLIOGRÁFICAS}

Agamben, Giorgio. Homo sacer. Valencia, España: Pre-textos, 2010.

Cordua, Carla. Explicación sucinta de la Filosofía del Derecho de Hegel. Santa Fe de Bogotá, Colombia: Temis, 1992.

Deleuze, Gilles. Foucault. Barcelona Espańa: Paidós, 2007.

Foucault, Michel. Historia de la sexualidad. La voluntad del saber. Buenos Aires, Argentina: Ediciones Siglo XXI editorial, 2008.

. Microfísica del poder. Madrid, Espańa: La Piqueta, tercera edición, 1992.

El orden del discurso. Barcelona, España: Tusquets, 2002.

Godoy, Óscar. "Analítica del poder: Michel Foucault”, en Estudios Públicos No 40., Santiago, Chile: Centro de Estudios Públicos, primavera 1990.

Hegel, G. W. F. Principios de la Filosofía del Derecho o Derecho Natural y Ciencia Politica. Barcelona, Espańa: Edhasa, 1999.

. Fenomenología del Espiritu. México, D. F.: Fondo de cultura económica, 1971.

Hobbes, Thomas. El Leviatán. España, Madrid: Sarpe, 1984.

Huicı, Vicente. Espacio, tiempo y sociedad. Madrid, España: Akal, 2007.

Kojève, A. La dialéctica del amo y el esclavo en Hegel. Buenos Aires, Argentina: La Pléyade, 1985. 